\title{
Comparison of seven portable Raman spectrometers: beryl as a case study
}

\author{
Jan Jehlička, ${ }^{a *} \oplus$ Adam Culka, ${ }^{a} \odot$ Danilo Bersani $^{b} \oplus$ and Peter Vandenabeele ${ }^{c}$
}

\begin{abstract}
In this paper, a series of beryl varieties with the accent on emeralds was investigated using seven portable Raman spectrometers equipped mainly with 785- and 532-nm excitation lasers. Additionally, one dual system and a new portable sequentially shifted excitation Raman spectrometer were applied. The advantage of using handheld instrumentation for investigations to be carried out outside the laboratory is well documented. For major part of beryls (emeralds and aquamarines), the most intense Raman bands are found at correct positions $+/-2$ to $4 \mathrm{~cm}^{-1}$ using all the instruments (with the exception of one). Unambiguous identification of beryls is ensured by obtaining the strong characteristic of Raman features (1070 and $686 \mathrm{~cm}^{-1}$ ) of the whole spectrum. Spectroscopic performance and differences existing between the instruments not only from the construction and ergonomic point of view are discussed. All the instruments tested EzRaman-I Dual (Enwave Optronics), RaPort (EnSpectr), FirstGuard (Rigaku), FirstDefender XL and FirstDefender RM (Thermo Scientific), Inspector Raman (Delta Nu) and Bravo (Bruker) can be used for common gemmological and mineralogical work in situ. Two instruments (the RaPort and the sequentially shifted excitation Raman spectrometer Bravo) allow recording excellent quality Raman spectra comparable with laboratory dispersive Raman microspectrometers. Copyright @ 2017 John Wiley \& Sons, Ltd.
\end{abstract}

Keywords: beryl; emerald; aquamarine; mobile Raman spectroscopy

\section{Introduction}

Raman spectroscopy in its different operative modes and instrumental settings is widely used in mineralogical and gemmological research. Lately, lightweight portable or handheld Raman spectrometers have developed to instruments that can be recommended for common geological fieldwork. The miniaturization of the instrumentation progressed, and lightweight as well as palmsize Raman spectrometers can nowadays be used for detection of unknown samples, including minerals under outdoor conditions. Raman spectroscopy can be proposed as a technique of field detection and identification of minerals $s^{[1-3]}$ and biomarkers ${ }^{[4]}$ and suggestion for application in planetary research and astrobiology areas. $^{[5-7]}$ Clearly, lightweight portable Raman spectrometers have proved to be excellent tools for identification of mineral phases and all kinds of geomaterials fast, onsite and non-destructively. Onsite means not only under museum/gallery/depository environments from where the specimens/artefacts cannot be moved to the labs. More importantly, only portable Raman spectrometers allow direct and fast identification of such compounds under really outdoor or direct outcrop conditions. Geoscience, archaeology and art are those fields necessitating such a modern and performant tools.

The approach of recording spectra of minerals for their mostly instantaneous unambiguous identification has been developed using especially near infrared diode lasers excitation $(785 \mathrm{~nm}){ }^{[1]}$ Critical issues of outdoor work were highlighted recently including diverse analytical issues (collection of scattered light, time of accumulation). ${ }^{[1]}$ Many black and dark minerals are not easy to be identified using the portable systems because of absorption of the radiation and/or fluorescence, also positioning and focusing of the laser beam onto small grains can be a limiting factor in some cases. $^{[1,8,9]}$ Potential of different miniaturized and mobile Raman systems in art was reviewed by Colomban ${ }^{[10]}$ and in the fields of art, geoscience and forensic by Vandenabeele et al. ${ }^{[11]}$ More recently, smaller and robust instruments appeared on the market, equipped also with less common 1064- or 532-nm excitation lasers. The 1064-nm system was evaluated for geoscience and forensic applications, confirming good performance. ${ }^{[12]}$ A light handheld Raman spectrometer (Rigaku) equipped with a 532-nm excitation laser was used for example to detect microbial pigments under outdoor conditions for halophiles research. ${ }^{[5]}$ Few studies showed until now new possibilities of direct application of portable Raman spectrometers to detect gemstones or other minerals mounted in historical artefacts: Torah shield with quartz, agates, emerald, pearls and corals ${ }^{[13]}$; an 18th century monstrance from Prague Lesser Chapucines Treasury with numerous diamonds, garnets, amethysts, emeralds ${ }^{[14]}$ and rubies; and numerous 17 th and 18th century Sicilian jewels (diamonds, garnets, amethysts emeralds and rubies) collected in the frame of the Messina regional museum. ${ }^{[15]}$

* Correspondence to: Jan Jehlička, Institute of Geochemistry, Mineralogy and Mineral Resources, Charles University, Albertov 6, 12843 Prague 2, Czech Republic. E-mail: jehlicka@natur.cuni.cz

a Institute of Geochemistry, Mineralogy and Mineral Resources, Charles University, Prague, Czech Republic

b Department of Mathematical, Physical and Computer Sciences, University of Parma, Parco Area delle Scienze 7/a, Parma 1-43124, Italy

C Department of Archaeology, Ghent University, Sint-Pietersnieuwstraat 35, Ghent B-9000, Belgium 


\section{Beryl and its varieties}

Beryl $\mathrm{Be}_{3} \mathrm{Al}_{2} \mathrm{Si}_{6} \mathrm{O}_{18}$, a hexagonal cyclosilicate with six-membered single rings, is the most common member of the beryl group (other minerals: bazzite, indialite, stoppanite and pezzottaite). Beryl occurs in pegmatites, hydrothermal veins as well as in metamorphic schists. Non-transparent beryls with yellowish, greenish coloration are not very rare minerals in these environments. However, transparent and bright-coloured beryls are considered as gemstones. These varieties of beryl are much less current in the geological record: green emerald, pale blue aquamarine, yellow heliodor, pink morganite or colourless goshenite. Emerald colour is an intriguing and appreciated aspect of this gemstone. Variations in composition of emeralds including presence of other traces as $\mathrm{Cr}^{3+}, \mathrm{V}, \mathrm{Fe}, \mathrm{Mg}$, $\mathrm{Mn}, \mathrm{Zn}$ or Ti are reasons of slight changes of colour and hue. ${ }^{[16]}$ These rare minerals are frequently used in jewellery and mounted in art objects since Medieval ages. This is due to the mechanical resistance (hardness), optical properties as well as aesthetic aspects of gemstone varieties of beryl.

From structural point of view, hexagonal rings are connected in beryl by the Be ions on tetrahedral sites and $\mathrm{Al}$ ions in octahedral sites. ${ }^{[17-19]}$ The six-membered rings are stacked vertically along the $c$-axis forming channels, where 'impurities' ( $\mathrm{Na}, \mathrm{K}, \mathrm{Cs}$ or $\mathrm{OH}^{-}$, $\mathrm{H}_{2} \mathrm{O}, \mathrm{CO}_{2}, \mathrm{CH}_{4}$ ) can be accommodated. ${ }^{[19-21]}$ Raman spectra were commonly used to learn about beryl and its varieties, to identify this mineral unambiguously. ${ }^{[16,22]}$ Thorough analysis of a series of cut gemstones was made by Bersani et al. ${ }^{[23]}$ also to learn about presence of impurities and $\mathrm{OH} / \mathrm{H}_{2} \mathrm{O}$ that can help in provenancing raw or cut gemstones. Additionally, it was demonstrated that Raman microspectrometry is also well adopted in search of micrometric solid inclusions to be used for provenancing of gemstones (and namely beryls) as well as potential discrimination of synthetic specimens or mounted gemstones. Barone et al. ${ }^{[24]}$ carried out an analytical comparative study to evaluate Raman microspectrometers and a portable instrument to learn about sapphires.

There is a clear need for a technique that enables us fast and unambiguous determination of gemstones mounted in complex artefacts or unaccessible jewels, i.e. those conserved in museums, depositaries or treasuries. The motivation of this comparative study is to evaluate the performance of different mobile Raman spectrometers to investigate a series of beryls and their gemstone varieties - examples of silicates. Here, we test instruments developed in the first decade of the 21 st century as well as those that represent recently introduced analytical products with important technological added value (the RaPort with a 532-nm excitation and the sequentially shifted excitation Raman spectrometer). How about the possibilities to learn about Raman spectroscopic features of similar beryl varieties or even different specimens of one variety using nowadays common portable instrumentation? Is the technique sufficient for very fast and non-destructive mode of analysing gemstones in situ? The main goal of the present work is to compare practical and spectroscopic aspects of several types of portable Raman spectrometers. Three handheld Raman spectrometers equipped with 785-nm diode lasers are evaluated, two systems with 532-nm lasers as well as sequentially shifted excitation Raman spectrometer. Additionally, a dual instrument - permitting measurements using alternatively both the red and green excitations - is tested. Comparisons are made of data collected on a series of beryl varieties, mainly emeralds and aquamarines. Critical aspects are highlighted to permit recommendations for future use of those analytical tools for mineralogical uses.

\section{Experimental}

\section{Samples}

In total, 17 specimens of beryl were investigated: colourless transparent beryl (one sample), colourless translucent beryls (two samples), bluish beryl (one sample) and gemstone varieties: emeralds (eight samples plus two synthetic emeralds) and aquamarines (three samples). Some of the specimens are raw, and others are cut; see Fig. 1(a) and (b). The description of samples is given in Table 1, and photographs are given in Fig. 1(a) and (b). Possible effects of foreign traces (dust particles, organics, and water) were evaluated during the first stage of testing of beryls. None of the specimens investigated showed spectroscopic evidence of the presence of such compounds and obtained spectra contain Raman features corresponding to beryl. The dimension of specimens from currently studied series ranges from $3 \mathrm{~mm}$ to about $42 \mathrm{~mm}$ in length. This was not limitative at all to be illuminated using diode lasers of portable instruments, and scattered light was successfully collected from the minerals as well.

\section{Instrumentation}

In total, seven different portable, more or less lightweight Raman spectrometers were used in this work (Fig. 2). They differ in detail in construction as well as in general shape and mode of use. The instruments can be divided into three groups based on the excitation wavelength: green laser $(532 \mathrm{~nm}$ ) is used in instruments EzRaman-I Dual (DG) by Enwave Optronics, Irvine CA, USA; RaPort (G) by EnSpectr, San José CA, USA and FirstGuard (F) by Rigaku, Wilmington MA, USA. Near-infrared laser $(785 \mathrm{~nm})$ is used in instruments FirstDefender XL (A) by Thermo Scientific, formerly Ahura, Wilmington MA, USA; FirstDefender RM (B) by Thermo Scientific, formerly Ahura, Wilmington MA, USA; Inspector Raman (C) by Delta Nu, Laramie WY, USA; EzRaman-I Dual (DR) by Enwave Optronics, Irvine CA, USA. Two excitation wavelengths (probably 785 and $1064 \mathrm{~nm}$ ) are used in instrument Bravo (E) by Bruker, Ettlingen, Germany. The details of technical specifications are summarized in Table 2.

The positioning of the samples and instruments is facilitated by the fact that the samples studied were of small dimensions (millimetres to centimetres), so rather than holding the instruments, the samples were held in the contact with the instruments while the instruments remained on the desk. This allows for the minimization in the changes of the positioning/focus in the sample/instrument system. All the 'pistol-like' instruments, i.e. Inspector Raman (C), RaPort $(G)$ and FirstGuard $(F)$ as well as the remaining handheld instruments, i.e. FirstDefender $X L(A)$, FirstDefender RM (B) and Bravo (E) are designed to measure in a direct contact of the sample and the instrument's head (zero working distance), which is typically a component made of plastic, sometimes removable, which purpose is to protect the laser optics and at the same time defines the original focal distance of the laser so that when the sample is analysed in a direct contact with the plastic head, the laser is ideally focused. For the EzRaman-I Dual instrument, the working distance was $3 \mathrm{~mm}$. From the ergonomics of the measurements, it is preferable that the instruments that are controlled by laptop computers be operated by two persons, one is holding the sample and the other operates the control program.

The reference spectrum of one specimen of colourless beryl from Habachtal was recorded with a Renishaw InVia Reflex microspectrometer (Laboratory Raman microspectrometer, 

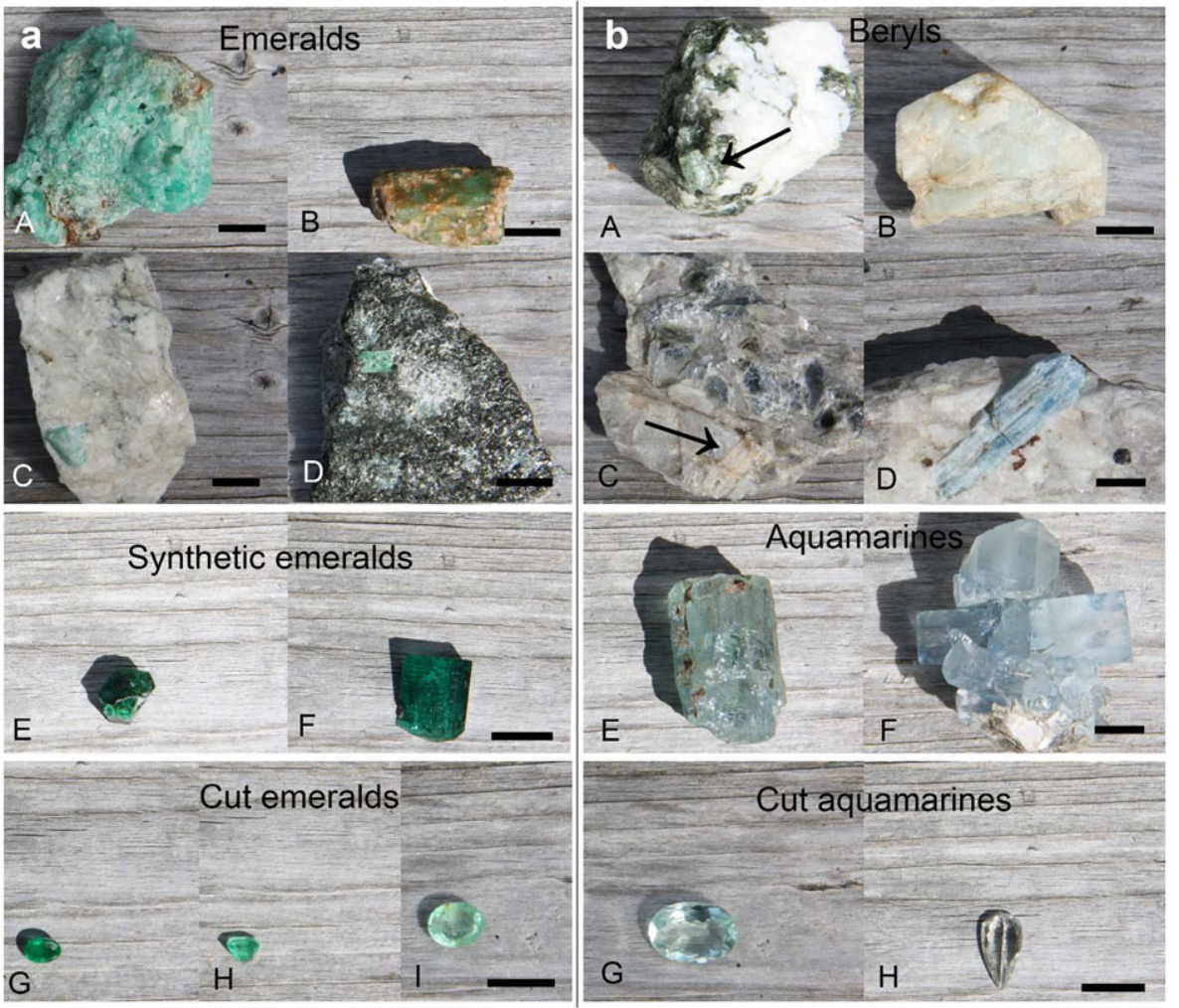

Figure 1. (a) Photographs of studied emerald samples. Natural emerald specimens: sample 1 (A), sample 7 (B), sample 9 (C) and sample 11 (D). Examples of synthetic emeralds: sample 5 (E) and sample 6 (F). Cut emerald gemstones: sample $3(\mathrm{G})$, sample 2 (H) and sample 12 (I). (b) Photographs of studied beryl and aquamarine samples. Beryl specimens: sample 10 (A), sample 13 (B), sample 16 (C) and sample 17 (D). Aquamarine specimens: sample 8 (E) and sample 15 (F). Cut aquamarine gemstones: sample $14(\mathrm{G})$ and sample $4(\mathrm{H})$. [Colour figure can be viewed at wileyonlinelibrary.com]

\begin{tabular}{|c|c|c|c|c|c|}
\hline Number & Mineral & Type of sample & Colour & Origin & Number/note \\
\hline 1 & Emerald & Massive crystalline mass & Light green, translucent & Chivor, Colombia & \\
\hline 2 & Emerald & Cut gemstone & Emerald green, transparent & Colombia & $0.46 \mathrm{ct}$ \\
\hline 3 & Emerald & Cut gemstone & Emerald green, transparent & Brazil & $0.47 \mathrm{ct}$ \\
\hline 4 & Beryl & Cut gemstone & Uncoloured, transparent & Brazil & $1.50 \mathrm{ct}$ \\
\hline 5 & Emerald & Synthetic, flux & Very dark green, transparent & - & \\
\hline 6 & Emerald & Synthetic, hydrothermal & Dark green, transparent & - & \\
\hline 7 & Emerald & Columnar crystal & Emerald green, barely translucent & Písek, Czech Republic & \\
\hline 8 & Aquamarine & Columnar crystal & Aquamarine bluish, transparent & Nissamia & \\
\hline 9 & Emerald & $\begin{array}{l}\text { Prysmatic crystal embedded } \\
\text { in pegmatite }\end{array}$ & Emerald green, non-translucent & Val Vigezzo, Alps, Italy & \\
\hline 10 & Emerald & Prysmatic crystal on schist & Emerald green, translucent & Val Vigezzo, Alps, Italy & \\
\hline 11 & Emerald & Small crystal on schist & Emerald green, translucent & Habachtal, Austria & \\
\hline 12 & Emerald & Cut gemstone & Emerald green, transparent? & Unknown (Istituto Gemmologico Ligure) & \\
\hline 13 & Beryl & Fragment of a big prismatic crystal & Pale bluish, not transparent & Piona (Colico), Lago di Como, Italy & \\
\hline 14 & Aquamarine & Cut gemstone & Bluish, transparent & Unknown (Istituto Gemmologico Ligure) & \\
\hline 15 & Aquamarine & Aggregate of prysmatic crystals & Bluish, transparent & Chumar Bakhur, Pakistan & \\
\hline 16 & Beryl & $\begin{array}{l}\text { Prysmatic crystal embedded in } \\
\text { pegmatite }\end{array}$ & White, opaque & Valchiavenna, Alps, Italy & \\
\hline 17 & Beryl & $\begin{array}{l}\text { Broken parallel aggregate of } \\
\text { prysmatic crystals }\end{array}$ & Blue, partly translucent & Valchiavenna, Alps, Italy & \\
\hline
\end{tabular}

$514.5 \mathrm{~nm}$ ) connected to a Leica optical microscope. Obtained Raman shifts were used mainly as a value to be compared with those obtained using portable systems. Excitation was provided by a 514.5-nm line of an argon gas laser. Accumulation time of $20 \mathrm{~s}$ for each scan and 20-30 scans were collected at laser power $\sim 20 \mathrm{~mW}$ to obtain a smooth Raman spectrum with maximum Raman bands visible. The spectra were recorded at a nominal spectral resolution of $2 \mathrm{~cm}^{-1}$, in the range of $100-1800 \mathrm{~cm}^{-1}$ and 


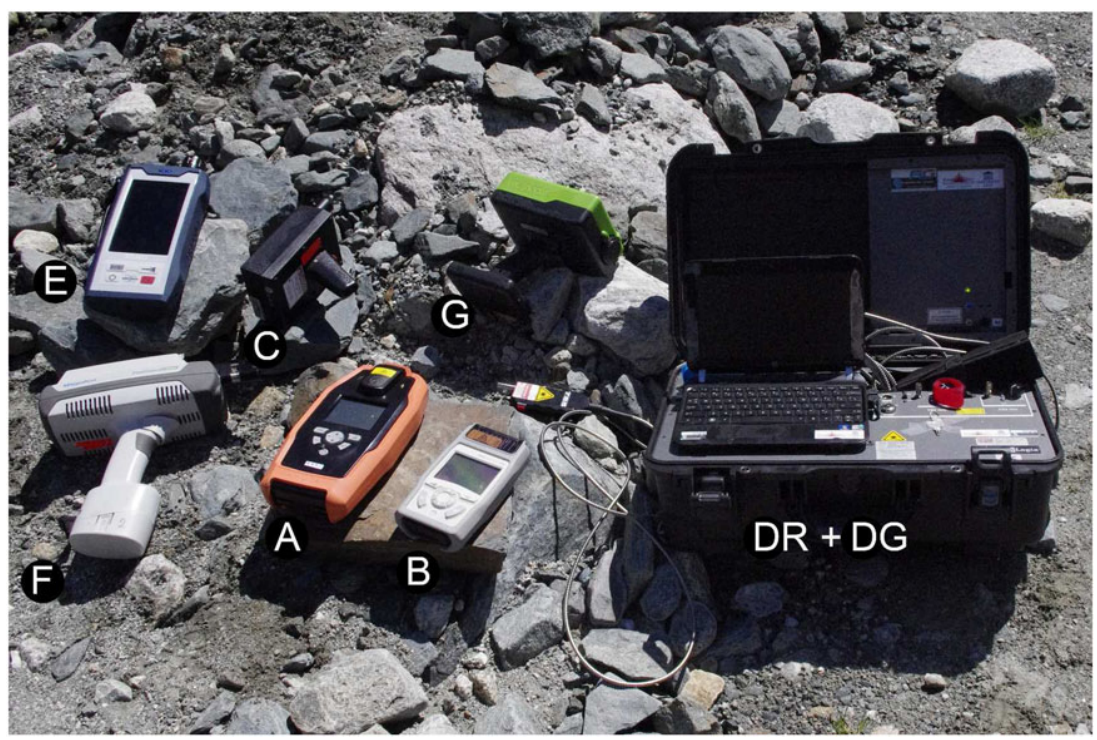

Figure 2. Miniature Raman spectrometers used in this study. FirstDefender XL (A), FirstDefender RM (B), Inspector Raman (C), EzRaman-I Dual using red laser (DR) and green laser (DG), Bravo (E), FirstGuard (F) and RaPort (G). [Colour figure can be viewed at wileyonlinelibrary.com]

$2800-3800 \mathrm{~cm}^{-1}$. All instruments provide export of Raman spectra into the Galactic.spc or in ascii format, easy to convert into .spc. Spectra were then worked with using GRAMS AI (Version 8.0, Thermo Electron Corp, Waltham, MA, USA). Raman spectra were not subjected to any data manipulation or processing techniques and are reported generally as collected. Typical data analysis times were 20-60 s, and multiple spectra were obtained for each compound; an optimization of the number of accumulations to improve the signal-to noise ratio is usually automatically carried out by the software.

Additionally, Raman spectra of eight selected beryl specimens were recorded also using two laboratory Raman microspectrometers with the same excitation as in the case of the portable instruments (532 and $785 \mathrm{~nm}$ ). In this case, the characteristic values of Raman spectra \{band positions and band widths [full widths at half maximum (FWHM)]\} obtained for the eight samples were confronted with those from portable instrumentation. For the 532-nm excitation, a Thermo Scientific DXR Raman Microscope interfaced to an Olympus microscope with a 50x long focus objective was used (Laboratory Raman microspectrometer, $532 \mathrm{~nm}$ ). Laser power was set to $10 \mathrm{~mW}$ (maximum), and the spectra were recorded using a high resolution grating in the range of $100-2000 \mathrm{~cm}^{-1}$. Shorter accumulation time for individual scan and a higher number of scans (10 s and 64 accumulations) were used. For the 785-nm excitation, a Renishaw InVia Reflex microspectrometer connected to a Leica optical microscope with a 50x long focus objective was used (Laboratory Raman microspectrometer, $785 \mathrm{~nm}$ ). Laser power was set to $\sim 100 \mathrm{~mW}$ (maximum), and the spectra were recorded at a nominal spectral resolution of $2 \mathrm{~cm}^{-1}$, in the range of $100-3800 \mathrm{~cm}^{-1}$. Accumulation time of $20 \mathrm{~s}$ was set for each scan, and 10 scans were collected.

Because this study was performed indoors, no additional shielding during the acquisition of the Raman spectra was deemed necessary. However, for the field work, the optimal solution that we have adopted is to use a piece of black cloth (synthetic, used by photographers) roughly $40 \times 40 \mathrm{~cm}$. This then serves as a cover for the spot that is being analysed as well as the head of the instrument or even the hand that is holding it. The need for this kind of shielding is variable; it is crucial during a sunny day; however, it can be recommended to shield always during the day.

\section{Results and discussion}

Beryl specimens of different types [Table 1; Fig. 1(a) and (b)] were analysed using the lightweight (handheld or portable) Raman spectrometers (Table 2; Fig. 2). The beryl samples can be divided into four distinct groups: A first group contains the green coloured emeralds (samples 1, 2, 3, 7, 9, 10, 11 and 12); a second group contains two representatives of the synthetic emeralds (sample 5 - flux emeralds and sample 6 - hydrothermal emerald); the third group contains aquamarines (samples 8, 14 and 15); finally, the fourth group contains transparent (sample 4), white (samples 13 and 16) and blue beryl specimens (sample 17). Some of the issues that will be discussed, such as fluorescence, have moderate to heavy influence on the resulting Raman spectra taken on the samples of green emeralds and synthetic emeralds while these interferences are very rarely observed for the aquamarines. The instruments can be divided into two groups based on the excitation wavelength they use. The Raman spectrometers that are using green excitation $(532 \mathrm{~nm})$ are EzRaman-I Dual (DG), FirstGuard (F) and RaPort (G); instruments FirstDefender XL (A), FirstDefender RM (B), Inspector Raman (C), EzRaman-I Dual (DR) use the near-infrared 785-nm excitation, and in this group can be placed also instrument Bravo $(E)$ that uses a combination of two lasers in the red to infrared region.

When studying emeralds with portable Raman spectrometers, different effects should be discerned. In the next paragraphs, we will evaluate the overall performance of the instruments, and in a first phase, we will use the number of observed Raman bands as a first-hand parameter for spectral quality. Secondly, the spectral calibration and band positions will be discussed. Thirdly, we will discuss the influence of fluorescence on our results. This is an effect that is rather important when studying emeralds. Moreover, when studying macro-crystalline phases, crystal orientation also influences the observed spectra. Finally, one of the instruments used 
(RaPort G) had the unique opportunity to be able to record Raman bands of high wavenumbers, which is useful when studying emeralds.

\section{Overall performance and number of observed beryl bands}

We present a summary of the acquired data for the Raman spectra in Table 3 and the analogous graphical representation in the Supporting Information as well as more detailed tables for each instrument and all the analysed samples. In Table 3, the total number of beryl bands detected in the spectrum around the correct position is given for each sample measured by all the instruments. It quickly presents the performance of individual instruments as well as the differences between the samples of beryls from different groups (emeralds, synthetic emeralds, aquamarines, etc.). Additional factors that have impact on the recorded spectra, such as excessive fluorescence, instrumental artefacts and excessive level of noise, are also indicated by a number of dashes (-); the more the signs, the more of these adverse effects were present in a spectrum. When a spectrometer was not able to record a spectrum for any reason, but most frequently due to the detector saturation from fluorescence, this is indicated by an (x). A spectrum containing a high number of beryl bands located at right positions as well as low amount of noise, fluorescence and artefacts is indicated by a $(+++)$ sequence. This last designation together with the total number of Raman bands can serve as an estimate of the performance of each instrument in a direct comparison. On this account, instruments EzRaman-I Dual (DR), EzRaman-I Dual (DG), Bravo (E) and RaPort $(G)$ performed better compared with the rest of the tested instruments, with instrument RaPort (G) having arguably the best performance. Additionally, this instrument operates on the spectral range of up to $4000 \mathrm{~cm}^{-1}$, which is unique among the tested instruments and allows for identification of $\mathrm{OH}$ stretching vibration of water molecules in channels in the beryl structure (Fig. 4). The position of the respective Raman bands can be influenced by the levels of alkaline ions ${ }^{[23,25]}$ and thus can serve for identifying the genesis of the emeralds (see further discussion). On the other side, the instrument FirstGuard (F) gave a poor performance even when analysing the most 'easy' samples such as colourless beryl or aquamarines and only the two strongest beryl bands could be detected in some spectra. Excessive artefacts of wave-like appearance (see Fig. S2 for instance) masked almost all the Raman signal. A relatively small number of detected Raman bands and the highest level of noise were observed in the spectra taken by instrument FirstDefender $\mathrm{XL}(\mathrm{A})$. Instrument Inspector Raman (C) performance was average, with a low number of both exceptionally good and bad spectra. Detailed tables containing all the Raman bands for all the samples as measured by all the eight portable instruments and two laboratory Raman microspectrometers are provided in Tables S1-S10.

Out of all measurements, two representatives of emerald and aquamarine samples were chosen based on the criterion that only these two samples provided Raman spectra when analysed by all instruments. Examples of Raman spectra taken on a lightly green coloured emerald (sample 12) can be seen in Figs S1 (red lasers) and S2 (green lasers). The spectra are given without any spectral manipulation covering the first order spectral region (200$1500 \mathrm{~cm}^{-1}$ ). The two strongest signature Raman bands of emeralds, located around 1069 and $687 \mathrm{~cm}^{-1}$, can be clearly seen in all the spectra in Fig. S1, but their relative intensity is reduced by a moderately steep background, typically rising towards the lower wavenumbers. The best spectra were recorded by instruments Inspector Raman (C), EzRaman-I Dual (DR) and FirstDefender RM (B). 


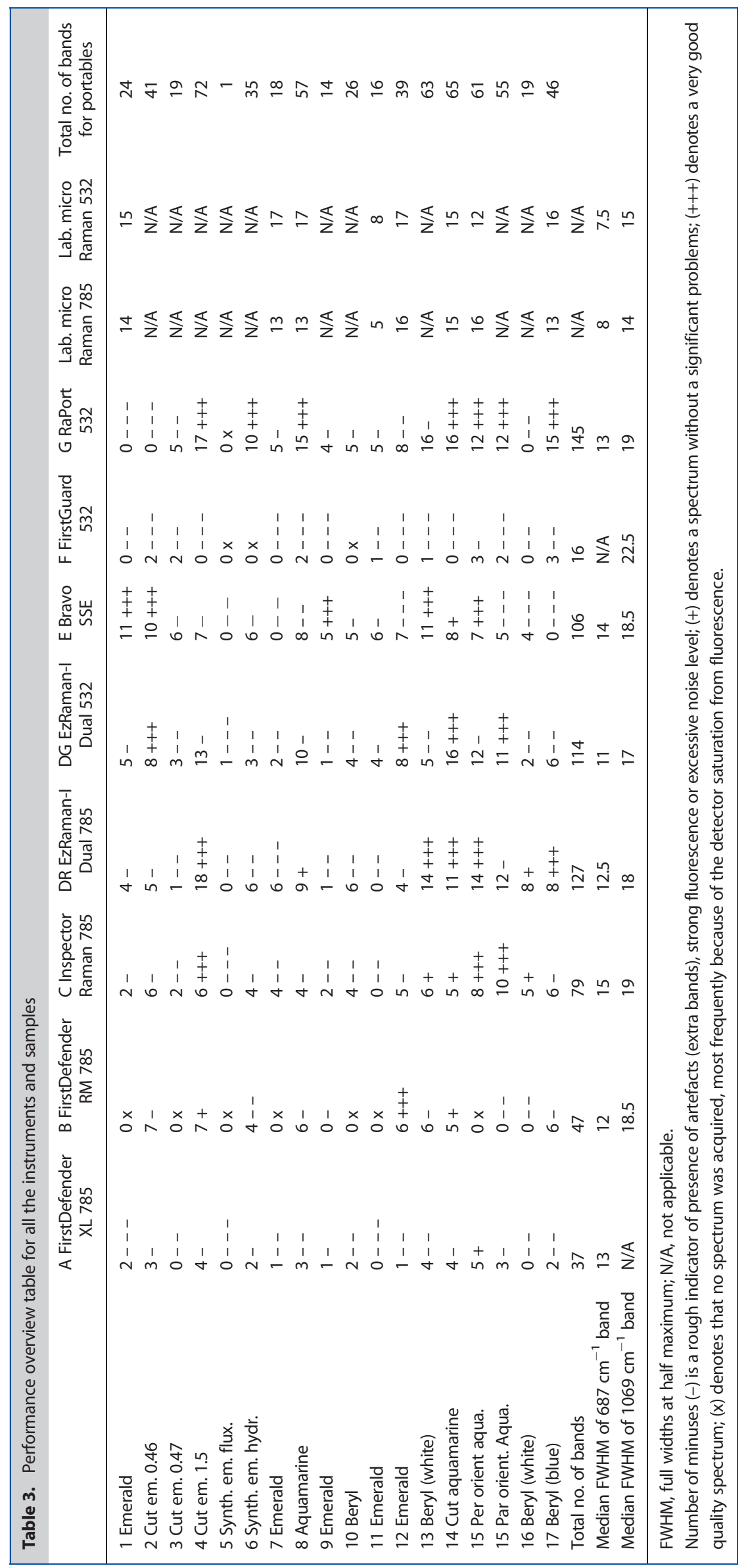


The spectrum recorded by instrument FirstDefender XL (A) yielded a very high amount of noise, where the strongest bands were only barely visible. The spectrum obtained by instrument Bravo (E) showed large number of artefacts that are probably caused by the fluorescence removal algorithm (see the next discussion). In Fig. S2, the spectrum recorded by instrument FirstGuard (F) shows a noisy, wave-like pattern and no Raman bands; the spectrum taken by instrument EzRaman-I Dual (DG) is of good quality and contains a moderate number of relatively strong bands and a smooth fluorescence background. The bottommost spectrum in Fig. S2 is recorded by instrument RaPort (G), and a strong fluorescence background and additional artefact bands can be seen in addition to the strong beryl bands.

Raman spectra taken on the aquamarine sample 8 are given in Figs S3 (red lasers) and S4 (green lasers). Although they contain a higher number of beryl bands of high intensity and generally a much lower amount of fluorescence background (compared with the spectra taken on sample 12), there are still obvious differences in the spectra taken by different instruments. The spectra taken by instruments Inspector Raman (C) and EzRaman-I Dual (DR) are of good quality, with the difference in the intensity of some band explained by a different orientation of the sample. The spectra taken by instruments FirstDefender XL (A) and FirstDefender RM (B) contain additional broad artefacts located at 1360 and $900 \mathrm{~cm}^{-1}$ and one very sharp artefact observed around $420 \mathrm{~cm}^{-1}$ in the spectrum taken by instrument FirstDefender RM (B). Again, instrument FirstDefender XL (A) provides a very noisy spectrum with only the three most intense aquamarine bands visible. The spectrum recorded with instrument Bravo (E) is of good quality; however, a slight deformation of the Raman bands can be observed. This effect is best visible on the $687 \mathrm{~cm}^{-1}$ band and could probably be related to the fluorescence removal process that was used to obtain this final spectrum, although with a much better result than what can be seen in Fig. S1. Very good quality spectra of the aquamarine sample were provided by instruments EzRaman-I Dual (DG) and RaPort (G), and again, a very bad quality spectrum full of wave artefacts and only the tiny peaks mark the supposedly strongest beryl bands in the spectrum taken by instrument FirstGuard (F).

A quite remarkable difference was observed between the two synthetic emerald samples. The flux-grown emerald 5 provided virtually no usable spectrum for any of the instruments, only a heavy fluorescence background that sometimes resulted in detector saturation and thus impossible measurement, while the hydrothermally produced emerald 6 was actually quite easy to analyse and the amount of the identified emerald band was relatively high (Fig. 3). This is interesting because the two synthetic samples both have a similar, rather dark green colour and a possible explanation could be that the flux emeralds contain some amount of the residual solvent material or may be additional chromophores used to correct the hue of the gem that contributes a major part of the fluorescence as can be seen in Fig. 4 (L).

\section{Shifts in band positions}

A major part of this study involves a determination of accuracy of the tested portable Raman spectrometers, i.e. how much the wavenumber values of recorded Raman bands of beryl samples differ from the reference values. Because in the experimental set were obtained not only samples with good or excellent Raman signal, such as colourless beryls, but mainly green coloured emeralds, only three signature (and also the strongest intensity) beryl Raman bands were selected: 1069, 687 and $398 \mathrm{~cm}^{-1}$, attributed to the $v(\mathrm{Si}-\mathrm{O})$,

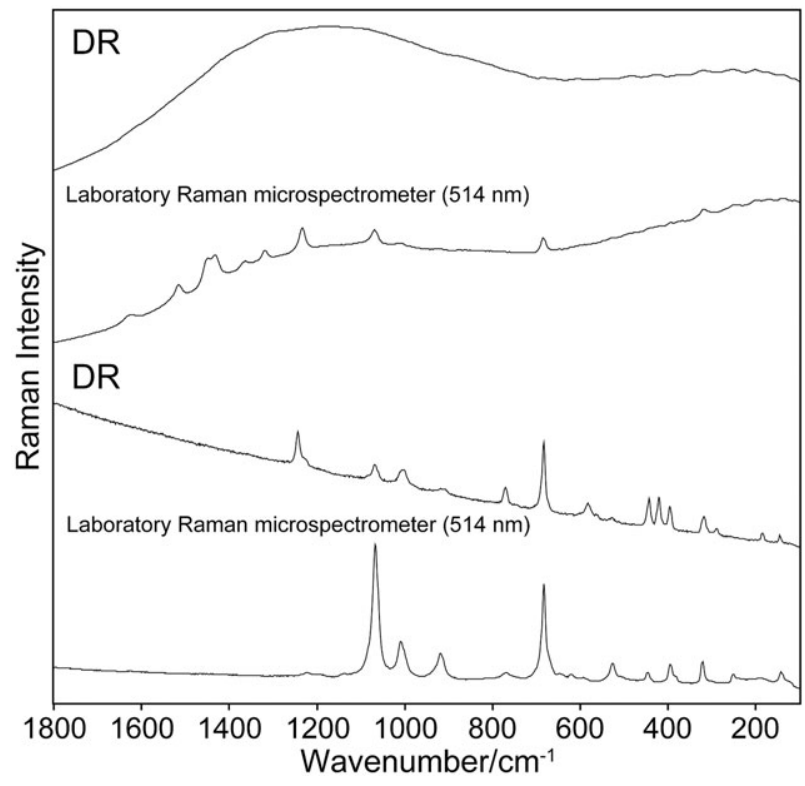

Figure 3. Raman spectra of synthetic emerald sample 5 recorded by instrument EzRaman-I Dual (DR) and by laboratory Raman microspectrometer $(514 \mathrm{~nm})$ and sample 6 recorded by instrument (DR) and by laboratory Raman microspectrometer $(514 \mathrm{~nm})$.

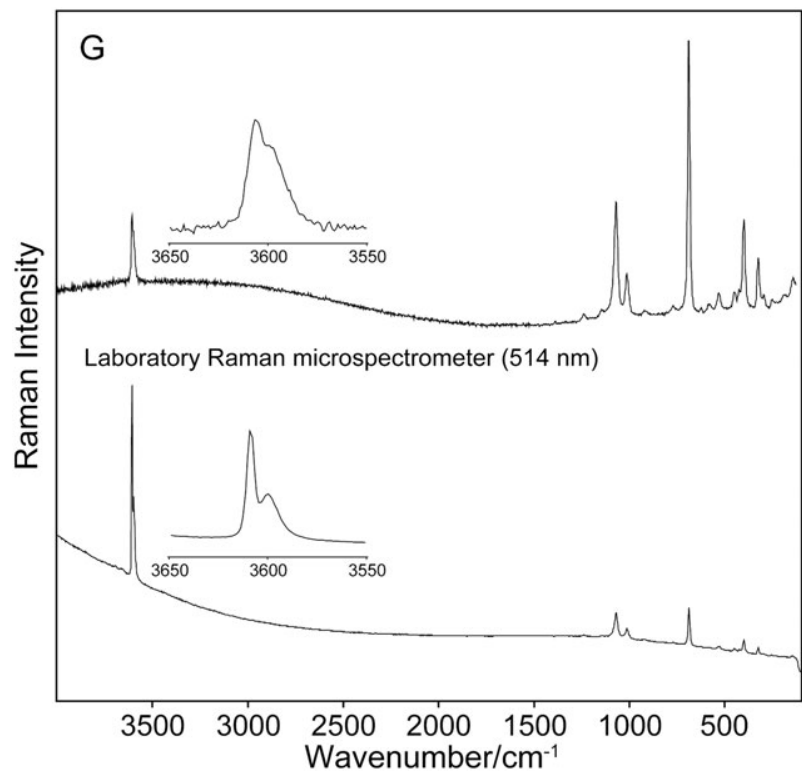

Figure 4. Raman spectra of cut aquamarine sample 4 recorded by RaPort (G) and by laboratory Raman microspectrometer (514 nm) including the region of $\mathrm{O}-\mathrm{H}$ stretching vibrations.

$v(\mathrm{BeO})$ and silicate ring vibration, respectively. These values were recorded on the sample of colourless beryl from Habachtal (sample not included in this study) using the laboratory Raman microspectrometer using the 514.4-nm excitation.

The results are summarized in the Fig. 5 in the following manner: All the 18 samples were analysed by all the instruments; for each analysis (Raman spectrum), the bands close to the three signature band positions $\left(1069,687\right.$ and $\left.398 \mathrm{~cm}^{-1}\right)$ were investigated for the shifts in position. Maximum number of total bands for all the samples was therefore $3 \times 18=54$. This number is given in Fig. 5 as a number $N$ for each instrument. Even by this simple but 


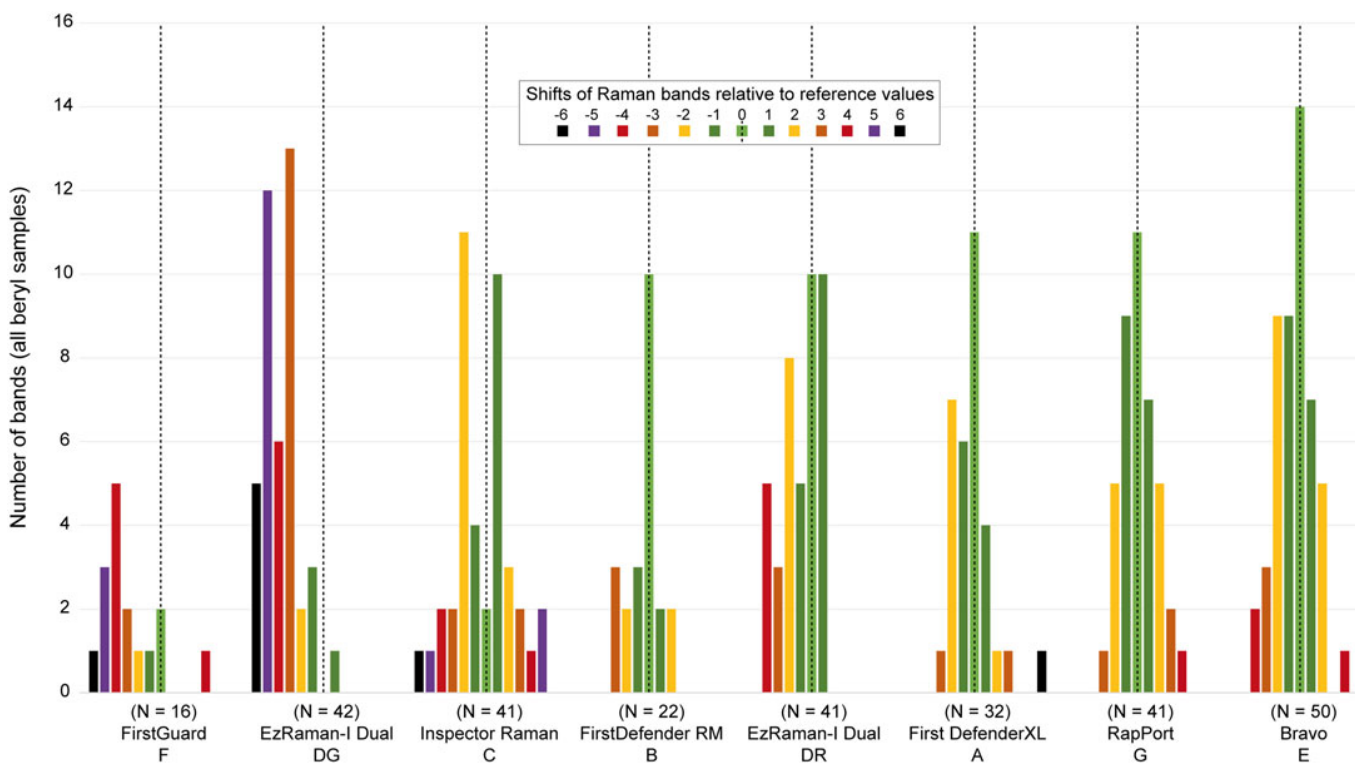

Figure 5. Shifts of Raman band positions of three signature beryl bands from values obtained with a laboratory Raman instrument $\left(1069,687 \mathrm{and} 398 \mathrm{~cm}{ }^{-1}\right)$ documenting the differences among the different portable Raman spectrometers. The numbers above the graph give a total number of Raman bands detected approximately at aforementioned wavenumber positions for all the studied samples for each instrument. [Colour figure can be viewed at wileyonlinelibrary.com]

important indicator, it is apparent that there have been major differences among the instruments. The two worst performing instruments in this evaluation were instruments FirstGuard $(F)$ and FirstDefender RM (B) that were able to detect only 16 or 22 out of 54 total possible bands, respectively. This was caused mainly by a high amount of noise or significant wave-like artefacts in the spectra taken by instrument FirstGuard (F), which often effectively masked even the strongest beryl bands. On the other end, the best performing instrument Bravo (E) identified 50 out of 54 possible bands, and instruments Inspector Raman (C), EzRaman-I Dual (DG), EzRaman-I Dual (DR) and RaPort (G) identified 41 to 42 bands.

For each instrument, a histogram documenting the shifts from the reference values for all the identified bands is given in Fig. 5 . The band shifts are marked in colour: the ideal zero shift is denoted by a light green, shifts of \pm 1 by darker green and shifts of \pm 2 by yellow. It is therefore apparent that all instruments except for the instruments FirstGuard (F) and EzRaman-I Dual (DG) are quite precise, with only a small fraction of detected bands shifted 3 and more $\mathrm{cm}^{-1}$ from the reference values. Furthermore, especially for the instruments RaPort $(\mathrm{G})$ and Bravo $(\mathrm{E})$, the distribution of shifts is close to the normal even for the high amount of total detected bands. Thus, the precision of instruments FirstDefender RM (B), EzRaman-I Dual (DR), FirstDefender XL (A), RaPort (G) and Bravo (E) is very good, with more than $60 \%$ of identified bands located at precise position or shifted only by $\pm 1 \mathrm{~cm}^{-1}$, and this is an excellent precision for the portable instruments. Still a good performance was recorded for the instrument Inspector Raman (C), where almost $80 \%$ of bands positions were in the interval of shifts $<-2,2>$. For the instrument EzRaman-I Dual (DG), the distribution of shifts is offset towards lower wavenumber by about $4 \mathrm{~cm}^{-1}$. This offset could probably be rectified via a post-calibration of the spectra, but this was not allowed in this test, because only the final spectra were analysed and directly compared for all the instruments. The instrument FirstGuard (F) had the worst performance, the lowest number of identified beryl bands. Their positions were shifted from the ideal value mostly towards lower wavenumbers but also with a significant spread. This is consistent with overall bad quality of spectra from this instrument, the amount of noise and artefacts. To sum it up, the precision was very good for the instruments FirstDefender RM (B), EzRaman-I Dual (DR), FirstDefender XL (A), RaPort $(G)$ and Bravo $(E)$, good for instrument Inspector Raman $(C)$ and instruments FirstGuard (F) and EzRaman-I Dual (DG) experienced a large negative shift (about $6 \mathrm{~cm}^{-1}$ ) from the correct positions.

Several samples of beryl have been additionally analysed using the laboratory Raman microspectrometers for comparison purposes. The laboratory instruments used the 532- and 785-nm excitation, which is the same as the excitation used by all the portable Raman spectrometers except Bravo (E). Examples of Raman spectra of emeralds and aquamarines are reported in Figs S5 and S6, and the positions of the bands of these samples are given in Tables $\mathbf{S 9}$ and S10. These tables together with Table 3 also list the total number of beryl Raman bands that were detected and their bandwidths (FWHM). As expected, the bandwidth of all the beryl Raman bands in the spectra taken with the laboratory instruments is significantly lower than in the spectra taken with the portable instruments. FWHM values for the band located at around $685 \mathrm{~cm}^{-1}$ are between 7 and $8 \mathrm{~cm}^{-1}$ for both the spectra taken with laboratory instruments, while the same parameter is in the range $11-15 \mathrm{~cm}^{-1}$ for the portable instruments' spectra. For another signature beryl band located at around $1069 \mathrm{~cm}^{-1}$, these values are 14-15 (laboratory instruments) and $17-22.5 \mathrm{~cm}^{-1}$ (portable). The spectra taken with portable instruments have in general significantly higher FWHM values of the beryl Raman bands when compared with the spectra taken with laboratory instruments. However, this was not a key issue in our study, because the medium and higher intensity Raman bands of beryl are quite separated from each other. Obviously, the laboratory instruments with higher spectral resolution can provide more detailed information, when needed.

The total number of beryl bands detected by all instruments for individual beryl samples is given in Table 3. For emerald sample 1, approximately 14 Raman bands were detected in the spectra taken with laboratory instruments, while only a few of the strongest bands were detected with the portable instruments [with the 
exception of Bravo (E)]. For the emerald sample 11 (from Habachtal), even the laboratory spectra contained only 5 and 8 Raman bands of beryl, which is comparable with the performance of Bravo $\mathrm{E}$ ( 6 bands) and RaPort G (5 bands) instruments. For the aquamarine samples, the portable instruments were able to detect more Raman bands in general. The wavenumber positions of all the detected beryl bands for all the samples and all the instruments are given in Tables S1-S10. For instance, the positions of the three strongest Raman bands in the spectra of sample 8 taken by the laboratory instruments were 395 and 396, 684 and $1068 \mathrm{~cm}^{-1}$ and the corresponding Raman bands in the spectra taken with portable instruments are not shifted by more than $3 \mathrm{~cm}^{-1}$, which is considered to be a good agreement.

\section{Impact of fluorescence}

One of the most important issues that has to be dealt with when analysing emeralds is the occurrence of fluorescence hampering the detection of the Raman signal. Fluorescence is often encountered in Raman spectra of emeralds, and this was also the case for the spectra obtained by the portable instruments in this study. The fluorescence phenomenon is intrinsically connected with the causes of green colour of emeralds, both based on the substitution of some (up to a few \%) Al atoms with $\mathrm{Cr}$ atoms in the crystal structure. Chromium atoms contain partially filled $3 \mathrm{~d}$ orbitals with unpaired electrons and thus are changing the physical characteristics of their surrounding with respect to transmission and absorption of incoming light. The more aluminium atoms are replaced by chromium, the stronger this effect is, and therefore, the deeper the green colour of the emeralds. Generally speaking, all the tested portable instruments obtained good quality spectra of colourless or slightly blue or green coloured beryl minerals. For the emerald samples of deeper green hues, the effects of fluorescence became more prominent and the Raman spectra were distorted or masked by the fluorescence background of various shapes and intensities for different instruments. No clear pattern can be observed, and the shape of the fluorescence background is strongly influenced by the concrete instrument's signal processing. This problem is more evident in portable spectrometers, where fully automatized procedures are present, with few possibilities to customize the measurement set-up.

Examples of spectra of emeralds containing various degrees of fluorescence are given in Figs S1 and S2; the fluorescence background is moderate even in the spectra taken on sample 12, which is a cut slightly green coloured stone. One of the instruments, Bravo (E), is equipped with an automated fluorescence removal capability, so this was an interesting feature to test on the emerald samples of deeper green colour. The first tested emerald sample was a cut gemstone 3 from Brazil, and the second was a piece of synthetic hydrothermally produced emerald 6. From Fig. 6 (top part), it is apparent that when the fluorescence background has a smooth and non-complicated structure, the final spectrum that is a result of the fluorescence removal process applied on the three raw spectra at the top is very clean and with a completely flat baseline. All the Raman bands of emerald are located at correct wavenumber positions, ${ }^{[14]}$ and most importantly, no additional false bands are produced. This example is in a strong contrast to the Raman spectra obtained by the same instrument on the sample of synthetic emerald that can be seen in Fig. 6 (bottom part). The top three raw Raman spectra demonstrate the complex shape of the fluorescence background in this sample. The three most intense Raman bands of emerald $\left(1069,687\right.$ and $\left.320 \mathrm{~cm}^{-1}\right)$ are only hinted at by the shift

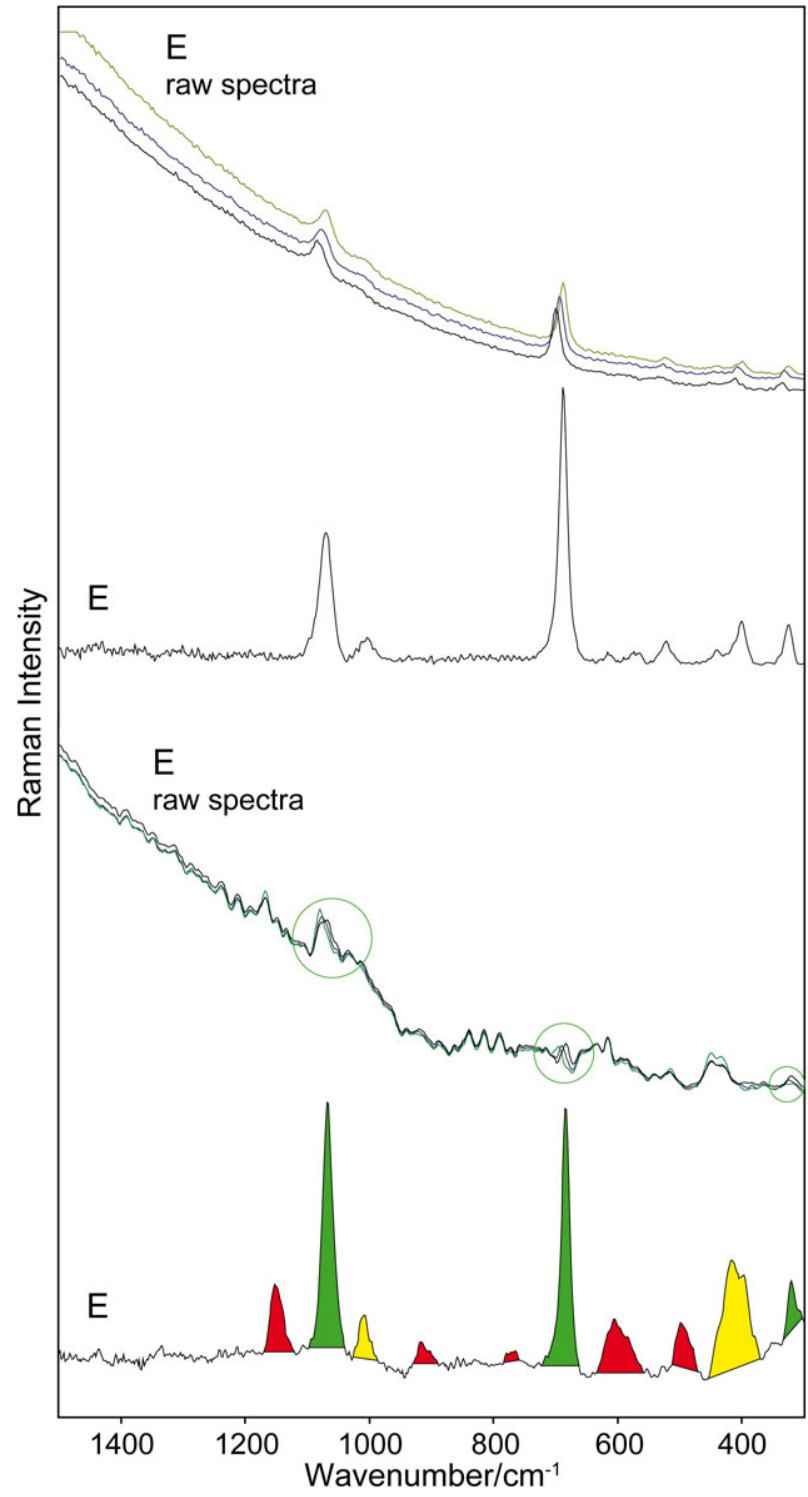

Figure 6. (Top) Raman spectra of sample 3 taken by instrument E. Top three are the raw spectra with the slight fluorescence background visible. All the beryl Raman bands are clearly distinguishable in the raw spectra. Bottom spectrum is a result of the fluorescence cancellation algorithm. (Bottom) Raman spectra of sample 6 taken by Bravo (E) instrument. Top three are the raw spectra with the heavy fluorescence background. The Raman bands (in circles) due to beryl (green) are almost concealed in the heavy fluorescence background. Bottom spectrum is a result of the fluorescence cancellation algorithm, and it can be clearly seen that this case results in the appearance of many false bands (red) that are relicts of this process. [Colour figure can be viewed at wileyonlinelibrary.com]

in their positions in the three different spectra, as opposed to the surrounding non-Raman features with unmovable positions, fluorescence related. The three bands are circled for a better orientation. That these spectra are a complicated task for the fluorescence removal process is apparent in the bottom spectrum in the Fig. 6. Only the three strongest Raman bands are located at the correct positions (marked in green), but additional false bands/features are formed at wavenumber positions where no emerald Raman bands can be located (marked in red). The bands marked in yellow are suspected to have a major contribution of the false bands (artefacts) although they are located at the positions where in general emerald bands can be observed. It is important to 
note that the shape and general appearance of the false extra bands are quite similar to that of the regular Raman bands, so this must be taken into account when the final spectrum is evaluated, and we strongly recommend to consult the raw spectra.

\section{Effect of crystal orientation}

Beryl tends to form well-developed columnar crystals, and the effects of different crystal orientation relative to the excitation laser on the resulting Raman spectra are well known. However, portable Raman spectrometers generally have inferior performance in comparison to the laboratory instruments, and while the impact of orientation of the crystal generally has a strong effect on the Raman spectra, several minor changes in the spectra might be a challenge for the miniaturized Raman instruments. Therefore, a section of this study is focused on establishing the ability of portable instruments to record the differences in the Raman spectra of oriented crystals. For this test, a superior quality aquamarine sample 15 (Fig. 1(b-F)) consisting of large crystals with well-defined smooth crystal faces was chosen. Two sets of measurements were acquired with each instrument; one set was oriented so the crystallographical c-axis was oriented along the laser beam that was pointed approximately at the centre of the hexagonal crystal pinacoid. The other set of measurements consisted of analysis of the aquamarine sample when the laser was pointed perpendicular to the $c$-axis and thus perpendicular to one of the six equivalent rectangular prism faces (Fig. 7). The two sets of Raman spectra demonstrate significant differences both in the intensity ratios of some Raman bands and disappearance of Raman bands (Fig. 7). The effect of orientation is so strong that only two Raman bands located at around 685 and $400 \mathrm{~cm}^{-1}$ ( $A_{g}$ modes) have similar intensities in both sets, while some bands are prominent in one orientation only. For instance, $1068 \mathrm{~cm}^{-1}$ band $\left(A_{g}\right.$ mode) is the strongest band by far in the perpendicular orientation and among weaker bands in parallel orientation. The reverse is true for the $320 \mathrm{~cm}^{-1}$ band. Bands located approximately at wavenumbers of 420,580, 1000 and $1240 \mathrm{~cm}^{-1}$ (all manifestations of the $E_{2 g}$ modes) are almost undetectable in the perpendicular

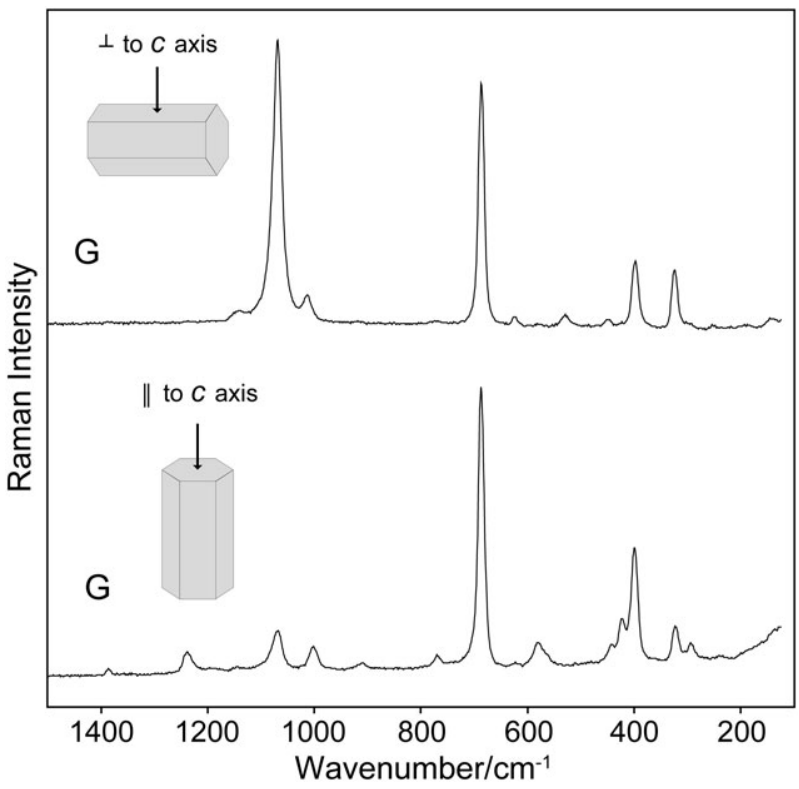

Figure 7. Raman spectra of oriented sample of aquamarine 15 recorded by RaPort $(G)$ instrument in orientation perpendicular to crystallographical caxis (top) and parallel (bottom). orientation and bands at 450,530 , and $1010 \mathrm{~cm}^{-1}\left(E_{1 g}\right.$ modes) in the parallel orientation. This strong dependence of resulting Raman spectra on the sample orientation was documented in the spectra taken with all instruments tested in this study. Especially good spectra obtained with instruments EzRaman-I Dual (DR) (Fig. S8), EzRaman-I Dual (DG) (Fig. S9) and Inspector Raman (C) (Fig. S10) as well as RaPort (G) allowed to observe interesting nuances such as changing intensities of the two very closely located bands: at about 1000 and $1010 \mathrm{~cm}^{-1}$, the former appearing in the parallel configuration only.

\section{Distinction among the high-alkali, low-alkali and synthetic beryls}

According to Bersani et al. ${ }^{[23]}$ using laboratory Raman apparatus, it is possible to divide emeralds (and in general beryls, including aquamarine) in two classes: low-alkali beryls (coming from lowalkali rocks such as granitic pegmatites or synthetized in water media) and high-alkali beryls (coming from high-alkali rock, like schists). Low-alkali beryls present an $\mathrm{OH}$ stretching peak related to type I water (at $3605 \mathrm{~cm}^{-1}$ ) more intense than the peak related to type II water (at $3595-3598 \mathrm{~cm}^{-1}$ ). In addition, the peak at nearly $1070 \mathrm{~cm}^{-1}$ in the low-alkali beryls is at wavenumbers lower than in high-alkali beryls (the discriminant wavenumber is $1069 \mathrm{~cm}^{-1}$ ). The same peak is sharper in low-alkali beryls than in high-alkali ones, being the 'threshold' width dependant on the used instrument. We wanted to evaluate if it is possible to obtain similar information also using portable instruments. We studied the spectra obtained with the instrument RaPort (G), the only one whose spectral range includes the $\mathrm{OH}$ stretching band. From the analysis of 13 beryls (excluding just the four with a very poor Raman signal), we found a perfect agreement with the previously stated rules. In all the samples, when the position of the $1070 \mathrm{~cm}^{-1}$ band is comprised between 1068 and $1069 \mathrm{~cm}^{-1}$, the bandwidth is $20 \mathrm{~cm}^{-1}$ or less. And, in these crystals, when the $\mathrm{OH}$ stretching band is visible (in 7 cases out of 13; in the remaining cases, the fluorescence was too high), the type I water peak at $3605 \mathrm{~cm}^{-1}$ was higher than that of type II water at $3598 \mathrm{~cm}^{-1}$. ${ }^{[15]}$ So these crystals behaved as lowalkali beryls (samples 4, 5, 6, 7, 8, 12, 13, 14, 15 and 16). In all the remaining samples $(3,9,10$ and 11$)$, we observed the opposite behaviour (peak position over $1069 \mathrm{~cm}^{-1}$, bandwidth larger than $20 \mathrm{~cm}^{-1}$, peak of water type II larger than the peak of water type I), as expected for high-alkali beryls. Looking at the (incomplete) data on the origin of the gems, it is possible to see that the crystals coming from the classic pegmatites lay in the 'low-alkali' group, while the freshly found 'Habachtal' emerald (sample 11) is in the high-alkali group, as expected, being embedded in a schist rock. These results can be confirmed (as regarding the behaviour of the $1070 \mathrm{~cm}^{-1}$ band) by the data obtained from the other instruments, with just small variations due to calibration details and the different resolution. A good agreement was found with the data obtained with the Bravo (E) instrument; for the samples 3, 10 and 11, the location of the main beryl band is in the interval of 1069.8 and $1070.7 \mathrm{~cm}^{-1}$ and bandwidths in the interval 23.5 and $25.9 \mathrm{~cm}^{-1}$, values typical for the high-alkali beryls. The positions of the $1070 \mathrm{~cm}^{-1}$ Raman band for low-alkali beryls were in the interval of 1068.7 and $1069.3 \mathrm{~cm}^{-1}$, and corresponding bandwidths were lower, in the interval of 17.9 and $22.6 \mathrm{~cm}^{-1}$. Band position and bandwidth for sample 6 , a synthetic hydrothermal emerald, were 1067.1 and $19.6 \mathrm{~cm}^{-1}$ using Bravo (E) instrument and 1068 and $17.8 \mathrm{~cm}^{-1}$ using the RaPort (G) instrument, respectively. These low values are lower than most of the values recorded on low-alkali 
beryls and may indicate a synthetic sample, according to Huong et $a l^{[26]}$ As an example, in the spectra obtained using the EzRaman-I Dual (DR) spectrometer operating at $785 \mathrm{~nm}$, the beryls subdivision is confirmed, using a threshold value of $1069.5 \mathrm{~cm}^{-1}$ for the peak position and $18 \mathrm{~cm}^{-1}$ for the bandwidth. The data obtained with all remaining instruments were of insufficient quality to perform this precision discrimination among the beryl types.

Two bands located at wavenumbers positions above that of Raman bands of emerald are the bands located at 1240 and $1387 \mathrm{~cm}^{-1}$. These bands can be detected in the spectra taken in the parallel orientation and are attributed to the Fermi doublet of vibrational bands of $\mathrm{CO}_{2}$ molecules trapped in the channels parallel to the $c$-axis in the beryl structure. Of these two coupled bands, the $1387 \mathrm{~cm}^{-1}$ band is attributed to the symmetric $v_{1}$ stretching vibration and $1240 \mathrm{~cm}^{-1}$ band is attributed to the first overtone of the $v_{2}$ bending vibration. ${ }^{[27]}$ Besides carbon dioxide, other small molecules are often trapped in the channels, most frequently the water molecules. And indeed, this is documented by the strong Raman signal in the $\mathrm{O}-\mathrm{H}$ stretching region $\left(3605 \mathrm{~cm}^{-1}\right)$ in the spectra obtained with the instrument RaPort $(\mathrm{G})$. This phenomenon of incorporation of $\mathrm{CO}_{2}$ and $\mathrm{H}_{2} \mathrm{O}$ molecules in channel cavities of other minerals such as cordierite has been studied with Raman spectroscopy for instance by Kolesov and Geiger. ${ }^{[28]}$

\section{Conclusions}

A series of portable Raman instruments tested in the frame of this study can be considered as an interesting collection of modern analytical tools to mainly be dedicated for an out-of-lab deployment. This is for the first time that experience obtained to evaluate the possibilities and limits for mineralogical work of seven individual instruments was collected. All the instruments tested can be used for common gemmological and mineralogical work - Raman spectra recorded contain the strongest features at correct expected positions. By this way, the instruments allow unambiguous identification of gemstones (here beryls). Very small instruments allow easy handling and highest portability [RaPort (G), FirstDefender (B)]. Two instruments: RaPort $(\mathrm{G})$ and Bravo (E) seem to allow recording excellent quality Raman spectra with band positions and intensities comparable with laboratory dispersive microspectrometers. These instruments were developed and introduced to the market quite recently (2015/2016). In these cases, excellent performance reflects modern concept and construction as well as novel approach and software for fluorescence suppression (Bravo E).

\section{Acknowledgements}

We would like to thank Tomáš Čapoun (Population Protection Institute, Lázně Bohdaneč), Pavel Matějka and Martin Člupek (UCT Prague) and Jan Neumann (Bruker Spectro).

\section{References}

[1] J. Jehlička, P. Vítek, H. G. M. Edwards, M. Heagraves, T. Čapoun, Spectrochim. Acta A 2009, 73, 410.

[2] J. Jehlička, P. Vítek, H. G. M. Edwards, M. Heagraves, T. Čapoun, J. Raman Spectrosc. 2009, 40, 1645.

[3] P. Vítek, J. Jehlička, H. G. M. Edwards, Appl. Spectrosc. 2013, 67, 767.

[4] A. Culka, J. Jehlička, L. Strnad, Spectrochim. Acta A 2012, 86, 347.

[5] J. Jehlička, A. Oren, J. Raman Spectrosc. 2013, 43, 1275.

[6] H. G. M. Edwards, I. Hutchinson, R. Ingley, J. Jehlička, Phil. Trans. R. Soc. A 2014, 372, 3205.

[7] J. Jehlička, A. Culka, L. Nedbalová, Astrobiology 2016, 16, 913.

[8] J. Jehlička, A. Culka, P. Vandenabeele, H. G. M. Edwards, Spectrochim. Acta $A$ 2011, 80, 36.

[9] P. Vandenabeele, J. Jehlička, P. Vítek, H. G. M. Edwards, Planet. Space Sci. 2012, 62, 48.

[10] P. Colomban, J. Raman Spectrosc. 2016, 43, 1529.

[11] P. Vandenabeele, H. G. M. Edwards, J. Jehlička, Chem. Soc. Rev. 2014, 43, 2628.

[12] P. Vítek, E. M. A. Ali, H. G. M. Edwards, J. Jehlička, R. Cox, K. Page, Spectrochim. Acta A 2012, 86, 320.

[13] K. Osterrothová, L. Minaříková, A. Culka, J. Kuntoš, J. Jehlička, J. Raman Spectrosc. 2014, 45, 830.

[14] J. Jehlička, A. Culka, M. Baštová, P. Bašta, J. Kuntoš, Phil. Trans. R. Soc. A 2016, 374, 20160042.

[15] G. Barone, D. Bersani, J. Jehlička, P. P. Lottici, P. Mazzoleni, S. Raneri, P. Vandenabeele, C. Di Giacomo, G. Larinà, J. Raman Spectrosc. 2015, 46, 989.

[16] I. Moroz, M. Roth, M. Boudeulle, G. Panczer, J. Raman Spectrosc. 2000, 31, 485.

[17] D. M. Adams, I. R. Gardner, J. Chem. Soc. Dalton Trans. 1974, 14, 1502.

[18] R. D. Aines, G. R. Rossman, Am. Mineral. 1984, 60, 319.

[19] C. Aurisicchio, G. Fioravanti, O. Grubessi, P. F. Zanazzi, Am. Mineral. $1988,73,826$

[20] J. Fridrichová, P. Bačík, V. Bizovská, E. Libowitzky, R. Škoda, P. Uher, D. Ozdín, M. Števko, Phys. Chem. Minerals 2016, 43, 419.

[21] B. A. Kolesov, C. A. Geiger, Phys. Chem. Miner. 2000, 27, 557.

[22] L. Kiefert, S. Karampelas, Spectrochim. Acta A 2011, 80, 119.

[23] D. Bersani, G. Azzi, E. Lambruschi, G. Barone, P. Mazzoleni, S. Raneri, U. Longobardo, P. P. Lottici, J. Raman Spectrosc. 2014, 45, 1293.

[24] G. Barone, D. Bersani, V. Crupi, F. Longo, U. Longobardo, P. P. Lottici, I. Aliatis, D. Majolino, P. Mazzoleni, S. Raneri, V. Venuti, J. Raman Spectrosc. 2014, 45, 1309.

[25] L. T.-T. Huong, T. Häger, W. Hofmeister, Gems Gemol. 2010, 46, 36.

[26] L. T.-T. Huong, W. Hofmeister, T. Häger, S. Karampelas, N. D.-T. Kien, Gems Gemol. 2014, 46, 287.

[27] B. A. Kolesov, C. A. Geiger, Amer. Miner. 2003, 88, 1364.

[28] B. A. Kolesov, C. A. Geiger, Amer. Miner. 2000, 85, 1265.

\section{Supporting information}

Additional Supporting Information may be found online in the supporting information tab for this article. 